\title{
Managing heart failure with preserved ejection fraction
}

\author{
Alexander Davidson ${ }^{1}$, Nivashinie Raviendran ${ }^{1,2}$, Charisma Nair Murali ${ }^{1,2}$, Phyo Kyaw Myint ${ }^{1}$ \\ ${ }^{1}$ Ageing Clinical and Experimental Research, University of Aberdeen, Scotland, UK; ${ }^{2}$ Royal College of Surgeon in Ireland-Perdana University, \\ Selangor, Malaysia \\ Contributions: (I) Conception and design: All authors; (II) Administrative support: A Davidson, N Raviendran, CN Murali; (III) Provision of study \\ materials or patients: None; (IV) Collection and assembly of data: None; (V) Data analysis and interpretation: A Davidson; (VI) Manuscript writing: \\ All authors; (VII) Final approval of manuscript: All authors. \\ Correspondence to: Alexander Davidson, MBChB, MRCP. Ageing and Clinical and Experimental Research Team, University of Aberdeen, Scotland, \\ AB25 2ZN, UK. Email: alexander.davidson1@nhs.net.
}

\begin{abstract}
Heart failure with preserved ejection fraction $(\mathrm{HFpEF})$ is increasing in prevalence as the general population ages. Poorly managed heart failure symptoms of decompensated HFpEF is one of the most common reasons for prolonged hospital admission. The high rate of morbidity and mortality associated with HFpEF is compounded by a poor understanding of the underpinning pathophysiology. Randomized controlled trials have so far been unable to identify an evidence base for reducing morbidity and mortality in patients with $\mathrm{HFpEF}$, although there is some evidence to support quality of life (QOL) improvement. In this review, we described the recent advances on the pathophysiological understanding of HFpEF, the current and emerging treatment strategies, and what this may mean for individual patients. Potential treatments for $\mathrm{HFpEF}$ were divided into their relative management strategies and the current evidence assessed for effect on HFpEF mortality, hospital admission frequency, and QOL improvement. Overall, the understanding of HFpEF pathophysiology is improving and has been made a priority in identifying potential therapeutic targets. There is growing evidence that patients with ejection fractions (EF) of less than $60 \%$ may obtain a mortality benefit from ACE-inhibitors, angiotensin-neprilysin inhibitors, Angiotensin Receptor Blockers, and Mineralocorticoid Receptor Antagonists. However, this covers only a small proportion of the HFpEF spectrum. Therefore, currently there are no universal treatment strategies recommended for HFpEF, and management should focus on an individualised approach and this should take into account the comorbidities of each patient.
\end{abstract}

Keywords: Heart failure; heart failure with preserved ejection fraction (HFpEF)

Submitted Jan 20, 2020. Accepted for publication Feb 07, 2020.

doi: $10.21037 / \mathrm{atm} .2020 .03 .18$

View this article at: http://dx.doi.org/10.21037/atm.2020.03.18

\section{Introduction}

Heart failure is one of the most common reasons for hospital admission in the elderly population, with increasing prevalence and extended hospital stays as the population ages, with $10 \%$ affected over the age of 80 (1). Approximately $50 \%$ of heart failure admissions are due to heart failure with preserved ejection fraction (HFpEF), formerly known as diastolic heart failure, with the overall general population prevalence between $1.1-5.5 \%$ (2). An optimal evidence-based strategy to manage heart failure in this patient group is unclear and is often complicated by comorbidities and polypharmacy (3). Echocardiogram (ECHO) characteristically demonstrates an ejection fraction (EF) $>50 \%$. As research has previously been focussed on EF $<40 \%$ [heart failure with reduced ejection fraction (HFrEF)], this precludes a HFpEF treatment strategy with a wellfounded evidence base (4).

As $\mathrm{HFpEF}$ is being increasingly recognised, HFpEF patients are now being targeted with novel treatments with the potential to improve symptoms, quality of life (QOL), reduce hospitalization and delay progression 

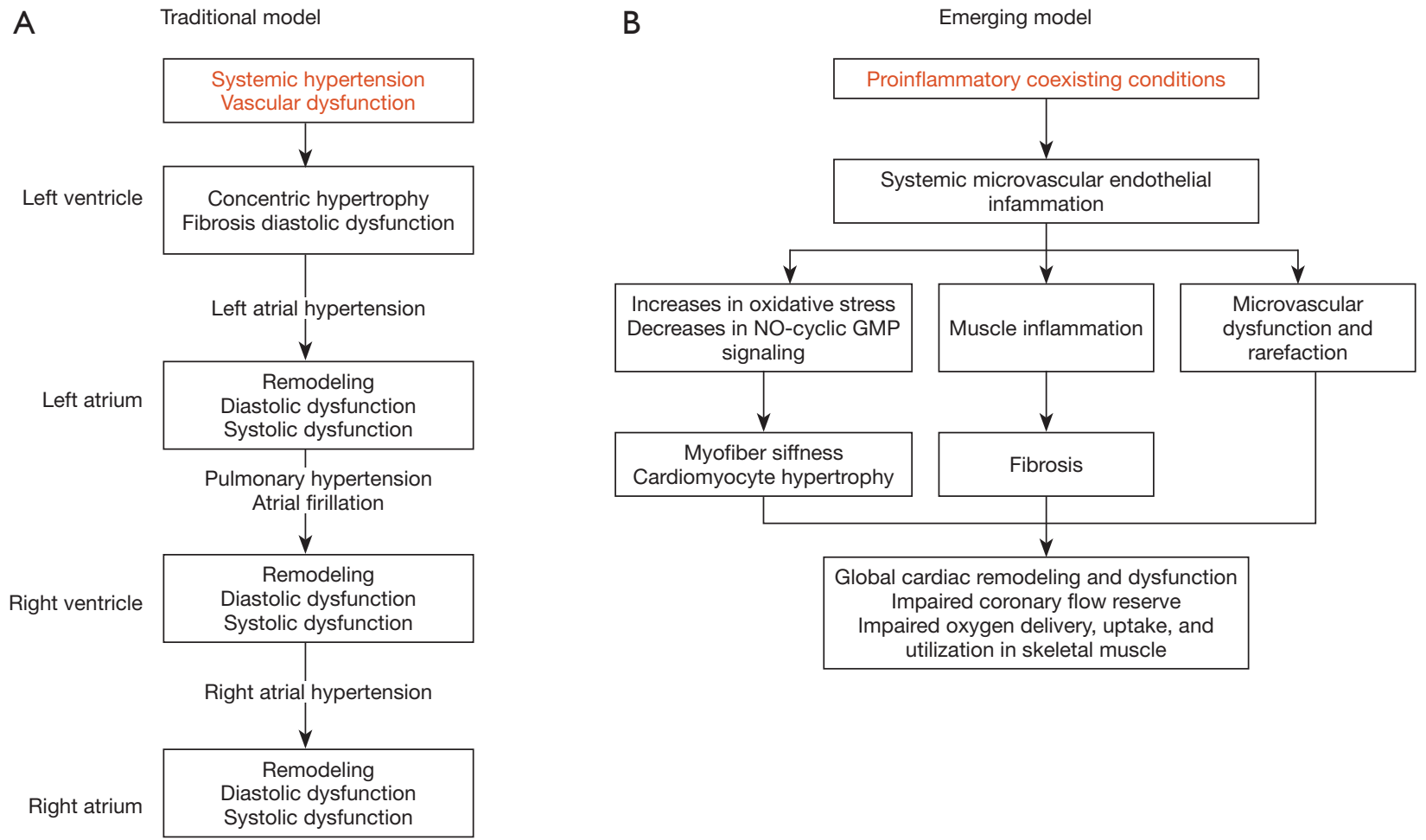

Figure 1 (A) Traditional and (B) emerging models describing pathology of HFpEF. Reproduced with permission from (Redfield et al.), Copyright Massachusetts Medical Society (5).

of the syndrome. Successfully improving treatment of this common syndrome is closely linked to the depth of understanding of the pathophysiology involved. This review describes the recent improvement in this understanding. We also discuss the current evidence base to support the treatment of HFpEF and present what the future holds with regard to large randomised controlled trials (RCTs).

\section{Methods}

Clinical trials and guidelines published since 2000 were analysed to assess the translational benefit of $\mathrm{HFrEF}$ therapies for HFpEF as well as de novo HFpEF trials. Studies were included in the review if data were reported for patients with $\mathrm{EF}>40 \%$, did not include congenital heart disease, and demonstrated evidence of diastolic failure on ECHO, which is synonymous with HFpEF.

\section{Pathophysiology}

Previously described as diastolic heart failure, HFpEF is a distinct functional deficit in heart function during diastole.
This is when the heart muscle contracts normally, hence normal ejection fraction, but does not adequately relax to allow normal filling during diastole. This results in an increased left ventricular end diastolic pressure (LVEDP), which is the hallmark of HFpEF. Despite the general understanding of HFpEF at a functional level, there are limited pathological targets for disease modification. The search for therapeutic targets has led to the development of the traditional and emerging pathological models of HFpEF (Figure 1) (5).

Historically, the traditional model explains that HFpEF stems from either systemic hypertension or vascular dysfunction which then further leads to concentric hypertrophy of $\mathrm{LV}$, diastolic dysfunction and fibrosis in the left ventricle (Figure 1A). This would cause left atrial hypertension, bringing about remodelling and both systolic and diastolic dysfunction in the left atrium. At this stage, atrial fibrillation and pulmonary hypertension can develop, leading to similar remodelling and dysfunction in the right side of the heart, throughout all of the cardiac cycle. This was demonstrated in 2019 and was most exaggerated during exercise states $(5,6)$. 
Table 1 Relative HFpEF criteria $(2,10)$

ESC criteria for HFpEF (in 2016)
NYHA associated HF symptoms
Elevated BNP and/or NT-proBNP
Echocardiography
LVH
LAE
LVEF $\geq 50 \%$
LVMI $>115 \mathrm{~g} / \mathrm{m}^{2}\left(>95 \mathrm{~g} / \mathrm{m}^{2}\right.$ females)
LAVI $>34 \mathrm{~mL} / \mathrm{m}^{2}$
E/e' ratio $>13$

AHA/HFSA-criteria for HFpEF (in 2017)

NYHA associated HF symptoms

Echocardiography

cLVH

LAE

LVEF $\geq 40 \%$

Evidence of LV diastolic dysfunction

AHA, American Heart Association; cLVH, concentric left ventricular hypertrophy; E/e', mitral inflow velocity/early diastolic velocity of mitral annulus; ESC, European Society of Cardiology; HFSA, Heart Failure Society of America; LAE, left atrial enlargement; LAVI, left atrial volume index; LVEF, left ventricular ejection fraction; LVH, left ventricular hypertrophy; LVMI, left ventricular mass index; NYHA, New York Heart Association.

The emerging model (Figure $1 B$ ) postulates that HFpEF is the reaction from coexisting proinflammatory conditions, i.e., obesity, hypertension, diabetes mellitus etc., giving rise to systemic microvascular endothelial inflammation. At the molecular level, these comorbidities cause reduction in the levels of nitric oxide (NO) and cyclic guanosine monophosphate (cGMP), cascading to events such as hypertrophy and increased stiffness in cardiomyocytes, fibrosis and vascular dysfunction. Ultimately, global cardiac remodelling and dysfunction take place together resulting in increased LVEDP (5).

Although treatment for $\mathrm{HFrEF}$ is similar regardless of heterogeneous aetiology (e.g., ischaemic vs. hypertensive pathology), the models described in Figure 1 illustrate specific targets that form part of a bespoke approach to $\mathrm{HFpEF}$, which also may require alterations according to phenotype and comorbidities. For example, in heart failure female sex is a non-modifiable risk-factor, but obesity may be a modifiable factor in the development of hypertension, and ultimately HFpEF (7). Therefore, it is probable that this individualised approach will be required, leading to difficulties in clinical trial design (8).

A study by Obokata et al. in 2017 aimed to demonstrate this via Echocardiography and invasive haemodynamic measurements during exercise. This study recruited 99 obese (BMI $>35 \mathrm{~kg} / \mathrm{m}^{2}$ ) and 96 non-obese (BMI $<30 \mathrm{~kg} / \mathrm{m}^{2}$ ) who had HFpEF, with 71 healthy non-obese volunteers as controls. Results showed that obese patients demonstrated increased in LV remodelling, plasma volume, biventricular filling pressures, and resultant RV dysfunction. In addition, increased heart volumes and pericardial fat deposition resulted in increased pericardial restraint, and therefore worsened diastolic filling (9). This was further supported by Parasuraman et al. who demonstrated that increased biventricular filling pressures, demonstrated by a flattened interventricular septum on ECHO, results in a reduced utilisation of the innate Frank-Starling mechanism in periods of exercise, leading to impaired diastolic function, and resultant reduced exercise tolerance (6). Thus, impaired diastolic filling of the left ventricle may have multiple aetiologies, each with a different solution. For example, whilst fluid overload requires diuretics, obesity may require supervised exercise regimes.

\section{Diagnosis of HFpEF}

HFpEF is defined as symptoms synonymous with heart failure resulting from impaired ventricular relaxation during diastole. Amongst other measurements, it manifests as increased LVEDP on ECHO.

Table 1 describes the criteria employed by the ESC and AHA/HFSA. The most significant difference is the cut-off for LVEF, with AHA/HFSA stating that LVEF greater than $40 \%$ with evidence of diastolic dysfunction is consistent with HFpEF, whereas, ESC dictates that LVEF should be greater than $50 \%$ with evidence of diastolic dysfunction, and LVEF of $40-49 \%$ are to be treated as a 'grey area', and essentially creating a buffer between the two diagnoses (10). Consequently, physicians and scientists have consistent difficulty in diagnosing HFpEF or designing optimal clinical trials due to different diagnostic criteria and variable patient phenotypes/comorbidities evident by different eligibility criteria observed in most of the HFpEF trials.

Other than LVEF, echocardiography can include multiple other parameters including LVMI, LAVI, and E/e' 
ratio, the latter of which replaces E/A ratio due to increased accuracy during assessment at rest, although E/e' is less favourable in concomitant systolic failure. However, it should be considered that the echocardiographic difference in LVEF in HFrEF and HFpEF even by the most stringent criteria is $10 \%$, which in a study in 2018 demonstrated that the inter-operator difference in measuring ejection fractions (EF) was between 0 and $10 \%$ with an average variation of $4.27 \%$. A difference that may increase in size when more complicated measurements are attempted (11).

A useful tool for physicians with limited access to invasive testing was developed by Reddy et al. This is a validated scoring tool (H2FpEF) to differentiate HFpEF from noncardiac causes of dyspnoea. This can direct the need for additional referrals/tests, as well as prompt the requirement to consider a different evidence base from HFrEF (12).

\section{HFpEF treatment strategies}

\section{Volume overload}

\section{Loop diuretics}

The administration of diuretics in the management of HFpEF aims to improve the symptoms of heart failure, namely oedema and dyspnoea. In 2008, the Hong Kong Diastolic Heart Failure Study involving 150 patients with LVEF $>45 \%$ who were randomised to either Diuretics alone, diuretics plus irbesartan, or diuretics plus ramipril. Outcome measures were QOL, 6-minute walk test, and echocardiographic measures of cardiac preload. This study found that the isolated use of diuretic was associated with a significant improvement of symptoms and QOL but had no mortality benefit or reduction in HF hospitalization. In addition, diuretics in combination with Irbesartan/ Ramipril marginally improved both LV systolic and diastolic longitudinal function, and lowered $\mathrm{N}$-terminal pro b-type natriuretic peptide (NT-proBNP) over a period of 1 year (13).

This was in agreement with a 2002 meta-analysis of RCTs of diuretic therapy in heart failure by Faris et al. Eighteen trials were included which showed a marked reduction in symptoms across the spectrum of EF (mean $46 \%)(\mathrm{P}=0.007)(14)$.

\section{Mineralocorticoid receptor antagonists (MRA)}

MRA's have an existing evidence base for treatment of HFrEF and demonstrate good symptom control and QOL improvement, some of which has recently been demonstrated in the TOPCAT trial, a trial which was designed to assess the efficacy of Spironolactone in HFpEF by the composite outcome of reduced CV mortality and cardiac arrest, with a secondary outcome of heart failure related hospital admissions. However, there has been no evidence to support the reduction of mortality/morbidity in HFpEF. Coupled with the undesirable side-effects such as hyperkalaemia, renal dysfunction, post-menopausal bleeding, and gynaecomastia, has resulted in MRA's falling out of favour (15).

The TOPCAT trial enrolled 3,445 participants worldwide who were randomised to treatment $(n=1,722)$ or placebo $(\mathrm{n}=1,723)$, with inclusion criteria modelled around the American Heart Association (AHA) definition of HFpEF. TOPCAT did not demonstrate improvement in the composite outcomes, but showed a small reduction in HF hospital admissions (NNT $=45)(15)$.

Subgroup analyses demonstrated a benefit in patients with elevated brain natriuretic peptide (BNP) (hazard ratio, $0.65 ; 95 \% \mathrm{CI}, 0.49$ to $0.87 ; \mathrm{P}=0.003)$. The authors speculate that this could be a chance finding or could be due to differences in baseline characteristics between these two strata. BNP has been shown to be associated with left ventricular remodelling and potentially an early marker of diastolic dysfunction. Therefore, BNP measurement may be an optimal feature in future study protocols $(15,16)$.

Of note, TOPCAT trial used less stringent AHA criteria, and post-HOC analysis indicated that only $34 \%$ of the included patients would meet the more stringent European Society of Cardiology criteria.

Kurrelmeyer et al. conducted a study with only 48 elderly female patients (age $>70$ ) and was powered for HF symptoms (NYHA class I-IV) and modification of 6-minute walk distance. Overall, the clinical composite score, encompassing NYHA level, and a rating on a 3-point Likert scale indicated an improvement with Spironolactone, however, no improvement in 6-minute walk distance was observed. This supports that Spironolactone stabilised the symptoms/clinical decline in this subset of elderly females. Although this study was not powered, Echocardiography also demonstrated improvement in transmitral Doppler early filling velocity to tissue Doppler early diastolic mitral annular velocity ratio (E/e') and therefore reduced filling pressures in the left ventricle. Furthermore, a reduction in the level of type III procollagen levels were observed, potentially indicating a reduction in myocardial fibrosis and resultant remodelling (17).

The currently ongoing SPIRRIT trial (due for 
completion in 2022) is powered to assess morbidity and mortality benefit of Spironolactone in HFpEF. The trial is recruiting 3,200 patients across Sweden and the USA with an elevated BNP, but also includes patients with moderately reduced $\mathrm{EF}$ at $40-49 \%$ (10).

\section{Rate control and management of $A F$}

\section{Beta-blockers}

Beta-blockers have long been demonstrated as having a positive benefit in HFrEF through reducing heart rate, reducing myocardial oxygen demand, improving diastolic filling time, as well as LV remodelling. However, there is minimal evidence to suggest that these benefits translate to HFpEF. Although there have been no large RCTs of betablockers in HFpEF patients, the OPTIMISE-HF registry in the USA to track the outcomes of 7,154 heart failure patients shed some light on the matter. Compared with the significant benefit for HFrEF, this study found that betablockers were not associated with any improvement in outcome for HFpEF (18).

A meta-analysis carried out in 2015 included 15 observational studies and two RCTs with a total of 27,099 HFpEF patients with EF $>40 \%$. Overall, the included studies were underpowered to significantly detect the endpoints (RR 0.94, $\mathrm{P}=0.718$ ). A random-effects model was utilised to assess the role of beta-blockers on allcause mortality and HF hospitalization. The study found no benefit in mortality ( $R R 0.94, P=0.718$ ) but significant benefit for HF hospitalisation (RR 0.81, P<0.001). Subgroup analysis also suggested that this effect was also limited to patients under 75 ( $\mathrm{RR} 0.92$ vs. $0.64, \mathrm{P}=0.81$ vs. 0.001) (19).

Recently Lam et al. (in 2018), reported the effect of beta-blocker use in heart failure patients with an ejection fraction $>50 \%$ and heart rate $>70$ beats per minute. A total of 4,537 patients were identified from the OPTIMIZE-HF registry with no contraindications for beta blockade, with 2,592 participants in the treatment arm; 730 patients in the treatment arm received high dose beta blockade (Atenolol $>100 \mathrm{mg}$, Carvedilol >50 mg, Metoprolol >200 mg, or Bisoprolol $>10 \mathrm{mg}$ ), and 1,740 received no treatment (205 returned no data). Follow-up was over 6 years (median 2.8 years) and the trial demonstrated a significant $14 \%$ risk reduction for combined all cause readmission and mortality $\mathrm{P}=0.027)(20)$.

Cleland et al. performed a meta-analysis of 11 clinical trials involving 17,312 patients evaluating all spectra of EF. Included in this analysis was 244 patients with EF $>50 \%$ where beta blockade provided no benefit in patients who were in either sinus rhythm or atrial fibrillation. However, this analysis lacked the power to detect a significant change for the HFpEF group but showed clinical improvements for $\mathrm{EF}<50 \%$ (21).

\section{Ivabradine}

Ivabradine is a rate control agent that acts on the $\mathrm{I}_{\mathrm{f}}$ (known as funny current channel) in the sino-atrial node and is only employed when the patient is in sinus rhythm. It can be used as an alternative option when patients are unable to tolerate beta blockade and has shown a reproducible benefit in HFrEF (22).

The EDIFY trial published in 2017 was a randomized, double-blind, placebo-controlled trial assessed whether heart rate $(\mathrm{HR})$ reduction with ivabradine improves cardiac function in HFpEF. The hypothesis was a reduction in heart rate would result in the reduction in LVEDP, as reflected by the E/e' ratio on echocardiography. Treatment was titrated until optimal HR of between 50-60 bpm was achieved. The combined primary endpoints were E/e' ratio, total distance on 6-minute walk test, and plasma NT-proBNP concentration, and these were measured at baseline, 2 months, and 8 months. Despite an average reduction in HR or $13 \mathrm{bpm}$, the study found no evidence of improvement of diastolic dysfunction or exercise capacity nor significant differences in adverse events (23).

\section{Digoxin}

Digoxin is a cardiac glycoside which exerts negative chronotropy and positive inotropy in the treatment of atrial fibrillation. It improves cardiac output, ejection fraction, and reduces filling pressures and pulmonary capillary wedge pressure. It is theorised that these effects may translate to a benefit in HFpEF.

Pau et al. (in 2019) evaluated the risk of all-cause mortality and composite outcome of mortality and HF admissions within 1 year. The study included 1,833 patients with HFpEF who were $>70$ years old and had EF $>50 \%$. The Digoxin group demonstrated a significant increased mortality (HR 1.46, $\mathrm{P}=0.001$ ) and $\mathrm{HF}$ admissions (HR 1.35, $\mathrm{P}=0.001$ ), this effect was pronounced at lower heart rates. Overall, Digoxin was shown to have a negative prognostic effect for HR $<90$, and a neutral effect on higher HR in patients with HFpEF (24). 


\section{Management of bypertension}

\section{Renin-angiotensin-aldosterone system (RAAS) inhibitors}

ACE inhibitors and ARBs are currently used to treat comorbidities such as chronic kidney disease (CKD) with proteinuria and also have a proven benefit in $\mathrm{LV}$ reverse remodelling in HFrEF.

The PEP-CHF trial was a placebo controlled RCT assessing 850 patients with $\mathrm{LVEF} \geq 40 \%$, with symptomatic $\mathrm{HF}$, and demonstrated diastolic dysfunction on echocardiography, were randomised to receive Perindopril or Placebo. After 2.1 years follow-up there was no statistically significant difference in all-cause mortality or cardiovascular hospitalisation $(\mathrm{P}=0.35)$. Poor recruitment and retention of study participants rendered this study with insufficient power to detect a difference in the primary endpoint (25).

The efficacy of ARBs was assessed in the I-PRESERVE (Irbesartan) and CHARM-preserved (Candesartan). I-PRESERVE enrolled 4,128 patients $>60$ years old, NYHA Class II-IV, and an EF $>45 \%$, who were randomly assigned to receive Irbesartan $300 \mathrm{mg}$ or placebo. The study failed to meet any of the primary endpoints $(\mathrm{P}=0.54)$. The CHARM study recruited 3,023 patients with LVEF $>40 \%$ and NYHA II-IV who were randomised to either Candesartan $32 \mathrm{mg}$ or Placebo. Candesartan showed a moderate reduction in HF hospitalisations $(\mathrm{P}=0.017)$ but no difference in the other endpoints $(26,27)$.

\section{Nitrate}

Nitrates are a regular feature in the management of angina, and heart failure which does not respond to conventional therapy. In HFpEF, a potential therapeutic target could be to reduce the bi-ventricular filling pressures by reducing cardiac preload. Additionally, animal models have suggested that nitrates may have a role in assisting ventricular relaxation during diastole, and therefore, a reduction in LVEDP (28).

The NEAT-HFpEF trial recruited 110 patients with HFpEF patients (LVEF >50\%) and compared placebo to Isosorbide Mononitrate (titrated from 30 to $60 \mathrm{mg}$, and then $120 \mathrm{mg}$ daily). Effect was assessed by activity levels, QOL scores, 6-minute walk distance, and levels of NT-proBNP, for all dosages. Activity levels were lower in the treatment group, and this effect worsened with increased dosage. There was no significant difference in the other outcomes. The 2017 Heart Failure Focussed Update from ACC/AHA/
HFSA suggested no advantage of adding nitrates to a heart failure treatment regime, a recommendation based on the results of this trial (29).

Targeted nitrate could potentially be used in HFpEF to increase exercise intolerance and is emerging as a possible target to optimize haemodynamics and reduce bi-ventricular interaction during stress. Borlaug et al. assessed 105 patients with $\mathrm{HFpEF}$ patients with $\mathrm{EF}>50 \%$ (mean $\mathrm{EF} 61 \%$ ), to assess whether inhaled Nitrite improved exercise tolerance, NT-proBNP levels, oxygen consumption, QOL score, and cardiac filling pressures (E/e'). A Nitrite compound was selected as it is more readily converted to the active Nitric Oxide in hypoxic/acidotic states and is converted in a 1-stage reaction leading to a rapid effect. This crossover trial randomised patients to 1 of 2 treatment groups (placebo first with crossover to nitrite, or vice versa). A washout period of two weeks was employed between crossover to ensure accurate data (30).

This study, however, failed to meet any of the end points, including a failure to reduce LVEDP as represented by E/ e' ratio. This was theorised to be in part due to the short acting nature of the nitrite compound, which is unable to achieve significant changes is cardiac function. In addition, the large percentage of obese patients in the trial (75\%) may indicate that the symptoms of HFpEF may be due to body habitus exerting a mass effect, rather than intrinsic changes in cardiac myocyte function (7). Further indicating the heterogeneity of $\mathrm{HFpEF}$, and the need to stratify patients according to comorbidities/phenotypes/genotypes to obtain accurate data (30).

Further clinical trials to assess the role of Nitrates in $\mathrm{HFpEF}$ are ongoing and are due to report in 2020. These include INDIE-HFpEF and KNO3CK OUT HFpEF trials.

\section{Calcium channel blockers}

There is minimal trial data available for the use of Calcium Channel Blockers (CCBs) assessing improvements in diastolic function and symptoms in HFpEF.

In 2014, Patel et al. performed propensity analysis on 1,620 patients obtained from the OPTIMIZEHF registry, who had a HFpEF and no prior history of CCB prescription. Two groups of 810 patients allocated to CCB treatment were matched for age, and average $\mathrm{EF}$ of $56 \%$. They were assessed for differences in HF/ non-HF hospitalisation rates, and all-cause mortality. No significant difference was found for all endpoints (HR: 1.05) (31). 


\section{Novel medications}

\section{Angiotensin-neprilysin inhibitor (Entresto)}

Entresto is the brand name for the Angiotensin-Neprilysin inhibitor which has demonstrated such efficacy in HFrEF, the PARADIGM-HF trial was halted early. This drug is a twice-a-day medicine that aims to improve overall cardiac function by enhancing the protective neurohormonal systems (natriuretic peptide system) while simultaneously inhibiting the harmful effects of the overactive reninangiotensin-aldosterone system (RAAS) (29). Other common heart failure medicines, namely angiotensin converting enzyme (ACE) inhibitors and angiotensin II receptor blockers (ARBs), only block the harmful effects of the overactive RAAS and have shown to assist LV remodelling. Entresto contains the Neprilysin inhibitor sacubitril and the ARB valsartan $(32,33)$.

In 2014, the PARADIGM-HF trial demonstrated superiority of Entresto vs. Enalapril alone for HFrEF with NYHA class II-IV symptoms. The benefits included a reduction in HF hospitalisations, as well as all-cause mortality. Physiologically, this resulted in a reduction in NT-proBNP in conjunction with LV/LA reverse remodelling and improved NYHA symptoms (32).

In response to the potential benefit to HFpEF patients, the PARAGON-HF randomized, double blind trial was completed in August 2019 and was the largest Phase III trial to date that aimed to demonstrate a similar benefit to HFrEF. Inclusion criteria of age $>50$, HFpEF (LVEF $>45 \%$ ), NYHA II-IV symptoms on diuretics, evidence of LAE or LVH on Echo and an associated elevation of NTproBNP. It demonstrated an improvement in NT-proBNP level and NYHA class at 12 and 26 weeks respectively. Additionally, it excluded any patient who had a previous $\mathrm{EF}<40 \%$ recorded at any time. A total of 4,822 patients randomised to Entresto or Valsartan and were followedup for 35 months on average. The study was powered at $95 \%$ to detect a $\mathrm{CV}$ death reduction of $10 \%$ and $\mathrm{HF}$ hospitalisation of $30 \%$, which was narrowly missed $(\mathrm{P}=0.06)$. Subgroup analysis demonstrated a potential positive effect for females (HR 0.73, 95\% CI: 0.59-0.70, $\mathrm{P}=0.06$ ), and LVEF <57\% (HR 0.78, 95\% CI: 0.64-0.95, P=0.03). No benefit was shown for EF $>57 \%$ (HR 1.00, 95\% CI: 0.81-1.23, $\mathrm{P}=0.02)$. In addition, the Entresto group had a reduced incidence of renal dysfunction (HR 0.50, 95\% CI: 0.33-0.77, $\mathrm{P}=0.002$ ) (34).

\section{Phosphodiesterase-5 inhibitors}

The PDE-5 inhibitor Sildenafil is an established treatment for management of primary pulmonary hypertension. It was postulated that by reducing pulmonary pressures, it would lead to reduced bi-ventricular interdependence and aid relaxation in diastole, resulting in reduced LVEDP. This is due to evidence of worsening outcomes in HFpEF with associated pulmonary hypertension and right ventricular failure secondary to increased afterload. It has also been suggested that Sildenafil may reverse cardiomyocyte hypertrophy where PDE-5 proteins are overexpressed in animal models $(35,36)$.

Marco et al. assessed $44 \mathrm{HFpEF}$ patients with pulmonary hypertension (PASP >40 mmHg), with HFpEF (LVEF $>50 \%$ ), and diastolic dysfunction were randomly assigned in a $1: 1$ ratio to either a placebo or sildenafil $(50 \mathrm{mg}$ tds). Measurements were made via invasive cardiac catheterisation for pulmonary pressures and RV function, and echocardiography to assess diastolic dysfunction. Follow-up at 12 months demonstrated improvement in hypertrophy, LV diastolic function, and reduced pulmonary pressures in the sildenafil group $(\mathrm{P}=0.01)$. There were no improvements in QOL outcomes (37).

RELAX trial involved $216 \mathrm{HFpEF}$ (EF >50\%) patients who were given Sildenafil after baseline invasive and echocardiographic measurements. Treatment effect was assessed at 24 weeks. Outcomes included effect on QOL, exercise capacity, left ventricular remodelling, diastolic function, and pulmonary artery pressures. At 24 weeks, there was no significant difference in exercise capacity or clinical status. There was also no significant difference in echocardiographic or cardiac MRI parameters. Notably, worsening of renal function in the treatment group was statistically significant, and this resulted in increases in NTproBNP, suggesting that the associated renal dysfunction was clinically significant (38).

Hoendermis et al. assessed 52 patients in a single centre RCT, with pulmonary hypertension secondary to HFpEF (EF $>45 \%$ ) who were randomised 1:1 to either Sildenafil $60 \mathrm{mg}$ or placebo. No significant benefit was demonstrated on pulmonary pressures, cardiac output, peak oxygen consumption, and adverse events were similar between the groups (39).

Overall, Sildenafil appears to demonstrate a small effect on echocardiographic and invasive measurements, but no effect on clinical status or QOL. 
Otbers

\section{Statins}

Statins are HMG-CoA Reductase inhibitors and are primarily used as lipid lowering therapies as part of ischaemic heart disease and stroke prevention. There have been no randomized clinical trials assessing the potential of Statin therapy in HFpEF, and this may be in part due to the drug being off-patent. However, several observational studies have demonstrated potential mortality benefit in this subset of patients.

In cardiac myocytes, statins inhibit guanosine triphosphate-binding protein and Rho-Kinase activity, theorised to be a key signalling pathway in ventricular remodelling post MI. Therefore, this effect of statins is independent to the cardiovascular risk modification related to LDL cholesterol. Suppression of LVH and LV remodelling was previously demonstrated in experimental models in mice (40).

Fukuta et al. (in 2005) studied a total of 137 patients in total with $\mathrm{EF}>50 \%$ were separated into groups who received statins $(n=68)$ and who did not $(n=69)$. After a mean follow-up of 21 months $( \pm 12)$, the statin group demonstrated a substantial improvement in survival (HR 0.22, 95\% CI: $0.07-0.64, \mathrm{P}=0.006)$. The patient numbers were small in this study, but baseline ECHO markers of diastolic function were near identical between the groups (41). This study was followed in 2015 as a meta-analysis of prospective observational studies with the use of propensity score analyses and included 4 studies ( 3 additional studies added to the Fukata 2005) and a total of 5,536 patients (2,768 on statins). The pooled analysis allied closely with the previous findings in that the statin group demonstrated a reduced mortality (OR 0.69, $\mathrm{P}=0.03)(41,42)$.

Marume et al. (in 2019), assess the efficacy of statin therapy in patients with HFpEF but in the absence of coronary artery disease (CAD). This study was based on the hypothesis that the main benefit of statins in HFpEF was the prevention of atherosclerosis and therefore a reduction in the ischaemia related dysfunction. A total of 414 with HFpEF without CAD were selected for propensity analysis and were selected from a nationwide HFpEF registry (JASPER registry). The statin group contained 81 patients with 333 in non-statin group, and follow-up was 25 months. The statin group showed improved all-cause mortality, noncardiac death, or $\mathrm{HF}$ rehospitalisation. In particular 3-year mortality was improved (HR 0.21, 95\% CI: 0.06-0.72, $\mathrm{P}=0.014)$. However, there was no significant difference in cardiac related deaths, and the mortality benefits of statins did not have a statistically significant interaction with cholesterol level or heart failure severity. In addition, the statin group had slightly higher echocardiographic indicators of diastolic failure at baseline (43).

\section{Non-pharmacological management}

\section{Cardiac rehabilitation}

Edelmann et al. considered exercise training to improve exercise capacity and diastolic function in patients with HFpEF. A total of 64 patients underwent 2:1 randomisation to receive supervised endurance and resistance training plus usual care $(n=44)$ against usual care alone. Primary endpoint was a change in peak VO2 (a measure of muscular oxidative function) at 3 months, with secondary endpoints being cardiac structure, diastolic function [E/e' and left atrial volume index (LAVi)], and QOL. This study demonstrated that exercise training improved exercise capacity, diastolic function and QOL with all measurements within 95\% CI range, $\mathrm{P}=0.001$. Improvement in these markers may be as a direct effect of exercise, or indirect benefits as a result of weight loss (44).

\section{Conclusions}

As evident by our findings, an effective pharmacological treatment that reduces morality in $\mathrm{HFpEF}$ remains elusive, with no RCTs demonstrating a clear mortality benefit for any drug class. Evidence provided in post-hoc subgroup analysis identified a potential mortality benefit for MRAs and RAAS inhibitors. Both the TOPCAT (Spironolactone) and CHARM (Valsartan) studies indicate a CV mortality benefit in LVEF $<60 \%$, with increasing benefit in the lower LVEF ranges. This finding extends to the PARAGONHF (Entresto) trial, where LVEF <57\% were associated with improvements in outcome. This may assist medication choice in HFpEF patients with concomitant fluid overload and/or hypertension, but only extends to a small fraction of the patient population with $\mathrm{EF}<60 \%$. A further RCT assessment of MRAs (SPIRRIT-HFpEF trial) is due for completion in $2021(8,15,34)$.

As statins are a less expensive and readily available medication which patients often already take, the analysis of statin trials offers an interesting treatment consideration other than cardiovascular or stroke risk. It also focussed on the pro-inflammatory 'emerging model' of HFpEF pathology. Three small trials assessing statins 
all demonstrated mortality benefits (HR 0.21, 0.22, and OR 0.69), although the trials had small patient numbers. Nitrates do not improve function or symptoms in $\mathrm{HFpEF}$ in the absence of limiting ischaemic heart disease, and although PDE-5 inhibitors demonstrate a small effect on echocardiography and invasive measurements, they have no effect on QOL or mortality (28-30,37-39,40-43).

Importantly, in all patient subgroups, the utility of maintaining mobility and regular exercise should not be understated and may improve diastolic heart function and QOL. There are no large-scale assessments of exercise therapy, but small trials have shown a clear benefit when compared to standard HF care (44). This approach may be strongly beneficial in particular phenotypes $(7,9)$.

Also, in comparison with HFrEF, HFpEF patients are more likely to die from non-cardiovascular events (10). Thus, it is advisable to screen patients who are diagnosed with HFpEF for associated comorbidities and undertake an individualised approach to treatment. In addition, the frailest patients who already have a reduced QOL, an individualised goal-directed approach with a strong focus on symptom improvement may be the optimal strategy.

In personalising treatment, caution should be exercised in the use of diuretics and beta-blockers in the frail population, as excessively reducing preload can lead to $L V$ underfilling and resultant fall in cardiac output. Beta blockers are often employed in coexisting IHD or AF and can magnify this effect by inhibiting the chronotropic response and thus, significantly increasing falls risk or end organ damage.

Potential reasons for equivocal or failed trials include trial design (e.g., different demographics, aetiologies, comorbidities, response to therapies, poor recruitment, and high drop-out rate); there is no global consensus on the definition of HFpEF; there is limited stratification of subgroups in HFpEF patients in RCTs; the lack of a standardised approach to admitting patients for HF across different healthcare systems. For future trial design, it would likely be beneficial if $\mathrm{HFpEF}$ patients were subcategorized into more mechanistically homogenous, discrete genotypes, phenotypes, and aetiologies (i.e., race, age, genetic, HTN, DM, AF, COPD, valvular heart disease, frailty). This could be in the form of a carefully matched case-control design to ensure a relevant stratification of the genotype/phenotypes.

Although there is now a renewed push for high quality evidence to support the treatment of HFpEF, this must be done with a clear definition and a stringent trial design to ensure reliable results within these subgroups. Improvements in understanding the pathophysiology involved will improve the accuracy of results and may lead to novel treatment agents to ameliorate the high morbidity and mortality burden and supersede the need to assess existing therapies that have failed thus far to demonstrate benefit.

\section{Acknowledgments}

Funding: None.

\section{Footnote}

Conflicts of Interest: The authors have no conflicts of interest to declare.

Ethical Statement: The authors are accountable for all aspects of the work in ensuring that questions related to the accuracy or integrity of any part of the work are appropriately investigated and resolved.

Open Access Statement: This is an Open Access article distributed in accordance with the Creative Commons Attribution-NonCommercial-NoDerivs 4.0 International License (CC BY-NC-ND 4.0), which permits the noncommercial replication and distribution of the article with the strict proviso that no changes or edits are made and the original work is properly cited (including links to both the formal publication through the relevant DOI and the license). See: https://creativecommons.org/licenses/by-nc-nd/4.0/.

\section{References}

1. Upadhya B, Kitzman DW. Heart Failure with Preserved Ejection Fraction in Older Adults. Heart Fail Clin 2017;13:485-502.

2. Owan TE, Redfield MM. Epidemiology of Diastolic Heart Failure. Prog Cardiovasc Dis 2005;47:320-32.

3. Yancy CW, Jessup M, Bozkurt B, et al. 2017 ACC/AHA/ HFSA Focused Update of the 2013 ACCF/AHA Guideline for the Management of Heart Failure. J Am Coll Cardiol 2017;70:776-803.

4. Savarese G, Lund LH. Global Public Health Burden of Heart Failure. Card Fail Rev 2017;3:7-11.

5. Redfield MM. Heart Failure with Preserved Ejection Fraction. N Engl J Med 2016;375:1868-77.

6. Parasuraman SK, Loudon BL, Lowery C, et al. Diastolic Ventricular Interaction in Heart Failure With Preserved Ejection Fraction. J Am Heart Assoc 2019;8:e010114. 
7. Malden D, Lacey B, Emberson J, et al. Body Fat Distribution and Systolic Blood Pressure in 10,000 Adults with Whole-Body Imaging: UK Biobank and Oxford BioBank. Obesity 2019;27:1200-6.

8. Kelly JP, Mentz RJ, Mebazaa A, et al. Patient selection in heart failure with preserved ejection fraction clinical trials. J Am Coll Cardiol 2015;65:1668-82.

9. Obokata M, Reddy YNV, Pislaru SV, et al. Evidence Supporting the Existence of a Distinct Obese Phenotype of Heart Failure With Preserved Ejection Fraction. Circulation 2017;136:6-19.

10. Ponikowski P, Voors AA, Anker SD, et al. 2016 ESC Guidelines for the diagnosis and treatment of acute and chronic heart failure: The Task Force for the diagnosis and treatment of acute and chronic heart failure of the European Society of Cardiology (ESC)Developed with the special contribution of the Heart Failure Association (HFA) of the ESC. Eur Heart J 2016;37:2129-200.

11. Kengne C, Ouattara B, Angoran I, et al. Interobserver variability in estimation of the left ventricular systolic function of dilated cardiopathies: Ejection fraction and global longitudinal strain. Available online: https://www. sciencedirect.com/science/article/pii/S1878648017306171

12. Reddy YN, Carter RE, Masaru O, et al. A Simple, Evidence-Based Approach to Help Guide Diagnosis of Heart Failure With Preserved Ejection Fraction. Circulation 2018;138:861-70.

13. Yip GWK, Wang M, Wang T, et al. The Hong Kong diastolic heart failure study: a randomised controlled trial of diuretics, irbesartan and ramipril on quality of life, exercise capacity, left ventricular global and regional function in heart failure with a normal ejection fraction. Heart 2008;94:573-80.

14. Faris R, Flather M, Purcell H, et al. Current evidence supporting the role of diuretics in heart failure: a metaanalysis of randomised controlled trials. Int J Cardiol 2002;82:149-58.

15. Pitt B, Pfeffer MA, Assmann SF, et al. Spironolactone for Heart Failure with Preserved Ejection Fraction. N Engl J Med 2014;370:1383-92.

16. McMurray JJV, O'Connor C. Lessons from the TOPCAT Trial. N Engl J Med 2014;370:1453-4.

17. Kurrelmeyer KM, Ashton Y, Xu J, et al. Effects of Spironolactone Treatment in Elderly Women With Heart Failure and Preserved Left Ventricular Ejection Fraction. J Card Fail 2014;20:560-8.

18. Hernandez AF, Hammill BG, O'Connor CM, et al. Clinical effectiveness of beta-blockers in heart failure: findings from the OPTIMIZE-HF (Organized Program to Initiate Lifesaving Treatment in Hospitalized Patients with Heart Failure) Registry. J Am Coll Cardiol 2009;53:184-92.

19. Bavishi C, Chatterjee S, Ather S, et al. Beta-blockers in heart failure with preserved ejection fraction: a metaanalysis. Heart Fail Rev 2015;20:193-201.

20. Lam PH, Gupta N, Dooley DJ, et al. Role of HighDose Beta-Blockers in Patients with Heart Failure with Preserved Ejection Fraction and Elevated Heart Rate. Am J Med 2018;131:1473-81.

21. Cleland JGF, Bunting KV, Flather MD, et al. Betablockers for heart failure with reduced, mid-range, and preserved ejection fraction: an individual patient-level analysis of double-blind randomized trials. Eur Heart J 2018;39:26-35.

22. Ferrari R, Ford I, Fox K, et al. The BEAUTIFUL Study: Randomized Trial of Ivabradine in Patients with Stable Coronary Artery Disease and Left Ventricular Systolic Dysfunction Baseline Characteristics of the Study Population. Cardiology 2008;110:271-82.

23. Komajda M, Isnard R, Cohen-Solal A, et al. Effect of ivabradine in patients with heart failure with preserved ejection fraction: the EDIFY randomized placebocontrolled trial. Eur J Heart Fail 2017;19:1495-503.

24. Llàcer P, Núñez J, Bayés-Genís A, et al. Digoxin and prognosis of heart failure in older patients with preserved ejection fraction: Importance of heart rate. Results from an observational and multicenter study. Eur J Intern Med 2019;60:18-23.

25. Cleland JG, Tendera M, Adamus J, et al. The perindopril in elderly people with chronic heart failure (PEP-CHF) study. Eur Heart J 2006;27:2338-45.

26. Massie BM, Carson PE, McMurray JJ, et al. Irbesartan in Patients with Heart Failure and Preserved Ejection Fraction. N Engl J Med 2008;359:2456-67.

27. Yusuf S, Pfeffer MA, Swedberg K, et al. Effects of candesartan in patients with chronic heart failure and preserved left-ventricular ejection fraction: the CHARMPreserved Trial. Lancet 2003;362:777-81.

28. Rammos C, Hendgen-Cotta U, Totzeck M, et al. Impact of dietary nitrate on age-related diastolic dysfunction. Eur J Heart Fail 2016;18:599-610.

29. Redfield MM, Anstrom KJ, Levine JA, et al. Isosorbide Mononitrate in Heart Failure with Preserved Ejection Fraction. N Engl J Med 2015;373:2314-24.

30. Borlaug BA, Anstrom KJ, Lewis GD, et al. Effect of Inorganic Nitrite vs. Placebo on Exercise Capacity Among 
Patients With Heart Failure With Preserved Ejection Fraction: The INDIE-HFpEF Randomized Clinical Trial. JAMA 2018;320:1764-73.

31. Patel K, Fonarow GC, Ahmed M, et al. Calcium channel blockers and outcomes in older patients with heart failure and preserved ejection fraction. Circ Heart Fail 2014;7:945-52.

32. McMurray JJV, Packer M, Desai AS, et al. AngiotensinNeprilysin Inhibition versus Enalapril in Heart Failure. N Engl J Med 2014;371:993-1004.

33. Langenickel TH, Dole WP. Angiotensin receptorneprilysin inhibition with LCZ696: a novel approach for the treatment of heart failure. Drug Discov Today Ther Strateg 2012;9:e131-9.

34. Solomon SD, McMurray JJV, Anand IS, et al. AngiotensinNeprilysin Inhibition in Heart Failure with Preserved Ejection Fraction. N Engl J Med 2019;381:1609-20.

35. Takimoto E, Champion HC, Li M, et al. Chronic inhibition of cyclic GMP phosphodiesterase $5 \mathrm{~A}$ prevents and reverses cardiac hypertrophy. Nat Med 2005;11:214-22.

36. Moraes DL, Colucci WS, Givertz MM. Secondary pulmonary hypertension in chronic heart failure: the role of the endothelium in pathophysiology and management. Circulation 2000;102:1718-23.

37. Guazzi M, Vicenzi M, Arena R, et al. Pulmonary Hypertension in Heart Failure With Preserved Ejection Fraction. Circulation 2011;124:164-74.

38. Redfield MM, Chen HH, Borlaug BA, et al. Effect of

Cite this article as: Davidson A, Raviendran N, Murali CN, Myint PK. Managing heart failure with preserved ejection fraction. Ann Transl Med 2020;8(6):395. doi: 10.21037/ atm.2020.03.18
Phosphodiesterase-5 Inhibition on Exercise Capacity and Clinical Status in Heart Failure With Preserved Ejection Fraction: A Randomized Clinical Trial. JAMA 2013;309:1268-77.

39. Hoendermis ES, Liu LCY, Hummel YM, et al. Effects of sildenafil on invasive haemodynamics and exercise capacity in heart failure patients with preserved ejection fraction and pulmonary hypertension: a randomized controlled trial. Eur Heart J 2015;36:2565-73.

40. Hattori T, Shimokawa H, Higashi M, et al. Long-Term Inhibition of Rho-Kinase Suppresses Left Ventricular Remodelling After Myocardial Infarction in Mice. Circulation 2004;109:2234-39.

41. Fukuta H, Goto T, Wakami K, et al. The effect of statins on mortality in heart failure with preserved ejection fraction: a meta-analysis of propensity score analyses. Int J Cardiol 2016;214:301-6.

42. Fukuta H, Sane DC, Brucks S, et al. Statin Therapy May Be Associated With Lower Mortality in Patients With Diastolic Heart Failure. Circulation 2005;112:357-63.

43. Marume K, Takashio S, Nagai T, et al. Effect of Statins on Mortality in Heart Failure With Preserved Ejection Fraction Without Coronary Artery Disease Report From the JASPER Study. Circ J 2019;83:357-67.

44. Edelmann F, Gelbrich G, Düngen H, et al. Exercise Training Improves Exercise Capacity and Diastolic Function in Patients With Heart Failure With Preserved Ejection Fraction. J Am Coll Cardiol 2011;58:1780-91. 